\section{Experiencia del trasplante de microbiota fecal a través de colonoscopía en el tratamiento de la infección por Clostridium difficile recurrente}

\author{
RODRIGO QUERA ${ }^{1}$, PATRICIO IBÁÑEZ ${ }^{1}$, DANIELA SIMIAN ${ }^{2, a}$, \\ DANIELA RIVERA ${ }^{3, a}$, GUILLERMO ACUÑA ${ }^{4}$, RICARDO ESPINOZA ${ }^{4}$
}

\section{Fecal microbiota transplantation through colonoscopy for Clostridium difficile recurrent infection. Report of eight cases}

Background: Most cases of Clostridium difficile infection (CDI) respond to a standard course of antibiotics, however recurrent CDI is becoming common and alternative therapeutic strategies are needed. In this scenario, fecal microbiota transplantation (FMT) has been suggested. Aim: To describe the efficacy and safety of FMT for the treatment of recurrent CDI. Patients and Methods: Review of medical records of all patients with recurrent CDI treated with FMT between April 2013 and April 2017. Demographic and clinical data were abstracted including details of treatment prior to FMT, rate of FMT treatment success and clinical course during follow-up period. Telephone surveys were conducted to determine patient satisfaction. Results: Eight patients aged 19 to 82 years (six women) underwent FMT. They experienced a median of four previous episodes of CDI (range 3-8). The mean duration of CDI was 18 days (range 3-36) before FMT. All procedures were performed by colonoscopy. Effectiveness with one session of FMT was 100\%. During the follow-up period (median 24 months, range 7-55), two patients developed CDI, one of them after using antibiotics. Adverse events were reported in three patients. Two had bloating and one patient with Crohn's disease and a history of bacteremia had an episode of Escherichia coli bacteremia. All patients would use FMT again if necessary. Conclusions: FMT through colonoscopy appears to be a safe, effective and long-lasting therapy in cases of recurrent CDI.

(Rev Med Chile 2018; 146: 823-830)

Key words: Clostridium difficile; Colonoscopy; Fecal Microbiota Transplantation; Gastrointestinal Microbiome.

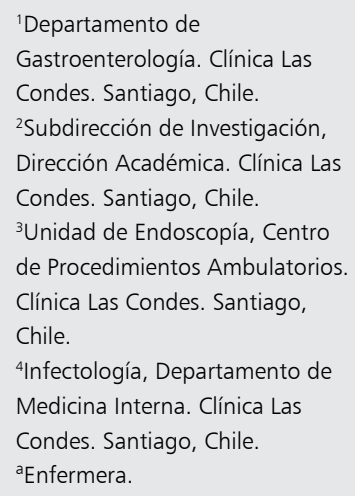

Los autores declaran no tener conflictos de interés.

Trabajo no recibió

financiamiento.

Recibido el 3 de noviembre de 2017, aceptado el 20 de julio de 2018.

Correspondencia a: Dr. Rodrigo Quera Teléfono: 226108048. Clínica Las Condes, Estoril 450. Santiago, Chile. rquera@clc.cl

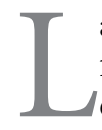

a infección por Clostridium difficile (ICD) fue descrita por primera vez en $1978^{1}$ y desde entonces ha sido señalada como la primera causa de diarrea nosocomial y la principal causa identificable de diarrea asociada a antimicrobianos $^{2}$. Su incidencia ha aumentado en las últimas décadas, asociándose a una elevada morbimortalidad y consumo de recursos sanitarios ${ }^{3,4}$. El tratamiento habitual de la ICD se basa en la suspensión del antibiótico asociado y el empleo de antibióticos como metronidazol, vancomicina, rifaximina y fidaxomicina ${ }^{5,6}$. A pesar de estas estrategias, la 
ICD se caracteriza por una alta tasa de recurrencia, que va desde $20 \%$ después de la primera infección hasta $65 \%$ luego de la segunda recurrencia ${ }^{7,8}$. Es en este último escenario donde se ha planteado el uso del trasplante de microbiota fecal (TMF) como una alternativa terapéutica, basada en la alteración de la diversidad bacteriana intestinal en pacientes con ICD recurrente $e^{5,9}$.

El TMF consiste en la administración de material fecal desde un individuo sano al paciente con el fin de restaurar la microbiota intestinal protectora ${ }^{10}$. Desde su primer reporte en $1958^{11}$, varios estudios han sido publicados en pacientes con ICD recurrente o refractaria, demostrando ser un procedimiento seguro con una efectividad que va entre 81 y 100\% ${ }^{12-17}$. Además, la recurrencia post-TMF es infrecuente, dado que la microbiota intestinal del donante se adapta rápidamente en el colon del receptor manteniéndose estable en el tiempo ${ }^{18}$. Estudios han sugerido que la administración vía colonoscopía es más eficaz ${ }^{13}$, segura ${ }^{19}$ y tiene mayor aceptación por parte del paciente ${ }^{20}$ al compararla con la administración vía nasoentérica o por enemas. En nuestro país existen tres publicaciones de casos clínicos sobre el uso de esta técnica, dos de ellos en pacientes con enfermedad inflamatoria intestinal ${ }^{21-23}$. Sin embargo, la efectividad o evolución a largo plazo de los pacientes post-TMF no ha sido reportada. Nuestro objetivo es mostrar la seguridad y eficacia a largo plazo del TMF realizado por colonoscopía en el tratamiento de la ICD recurrente.

\section{Pacientes y Métodos}

Estudio observacional donde se revisó la ficha clínica de todos los pacientes con ICD recurrente que fueron tratados con TMF en Clínica Las Condes entre abril de 2013 y abril de 2017. Todos los trasplantes fueron realizados por dos de los autores (RQ y PI) en el Centro de Endoscopía de nuestra institución. Los pacientes fueron incluidos considerando: a) el diagnóstico de ICD fundamentado por el cuadro clínico y la confirmación del Clostridium difficile por la técnica de PCR (reacción en cadena de la polimerasa) para toxina A y B; b) el antecedente de dos o más episodios demostrados de recurrencia de $\mathrm{ICD}^{27.8}$ tratados con esquema antibiótico estándar ${ }^{5,6,8}$; c) un seguimiento clínico de al menos 6 meses post-TMF. La gravedad de la ICD fue evaluada utilizando el índice de Hines
$\mathrm{VA}^{24}$. El seguimiento de los pacientes post-TMF se realizó mediante evaluación de la ficha clínica y encuesta telefónica de satisfacción. La respuesta al trasplante fue evaluada por la presencia de deposiciones formadas (escala de Bristol 3 o 4) a la semana del trasplante y hasta por lo menos tres meses posteriores a este.

\section{Protocolo de TMF}

Cada posible donante fue evaluado por gastroenterólogo (RQ o PI) e infectólogo (GA o RE) realizando una adecuada anamnesis y examen físico (Tabla 1). En aquellos que cumplieran con los criterios clínicos, se les solicitó exámenes de sangre y deposiciones según recomendaciones internacionales (Tabla 2). Cada paciente suspendió el tratamiento con vancomicina oral $24-48 \mathrm{~h}$ antes del trasplante y se preparó para la colonoscopía con polietilenglicol sin electrolitos (3-4 litros). El TMF se realizó con una muestra fresca de deposición del donante (obtenida no más de $6 \mathrm{~h}$ previo al trasplante).

Para la obtención de la solución de la microbiota se solubilizaron las heces del donante en suero fisiológico $(\mathrm{NaCl}$ 0,9\%) con una batidora de vaso destinada exclusivamente para el TMF. Una vez obtenida la solución homogénea, se filtró y se recogió el sobrenadante en jeringas de 60 $\mathrm{ml}$. Al realizar la colonoscopía y llegar al íleon se procedió a instilar la solución a través del canal de trabajo del colonoscopio siguiendo un esquema de retiro: $50 \mathrm{ml}$ en íleon, $150-300 \mathrm{ml}$ en ciego y colon ascendente, $50-100 \mathrm{ml}$ en colon transverso y los $50 \mathrm{ml}$ restantes en colon descendente. Durante el retiro del colonoscopio se evitó la aspiración para disminuir la posibilidad de retirar el material trasplantado. Una vez completado el procedimiento y el paciente recuperado de la sedación, se le dio loperamida $2 \mathrm{mg}$ por una vez, con el objetivo de retener el mayor tiempo posible la solución trasplantada (idealmente $6 \mathrm{~h}$ ).

Este estudio contó con la aprobación del Comité de Ética de la institución. Todos los pacientes firmaron un consentimiento para la realización de este procedimiento y autorizaron la publicación de los datos. A todos se les explicó previamente los beneficios y potenciales eventos adversos del TMF (relacionados con el procedimiento endoscópico y la presencia de síntomas digestivos postprocedimiento, tales como distensión abdominal y meteorismo). 


\section{Tabla 1. Criterios de selección para elegir al potencial donante (adaptado de referencia 8)}

Fármacos que puedan afectar la microbiota gastrointestinal. Excluir en caso de:

- Uso reciente (< 3 meses) de antibióticos, inmunosupresores, quimioterapia

- Uso crónico (> 1 mes) de inhibidores de la bomba de protones

Enfermedades gastrointestinales, metabólicas o neurológicas. Excluir en caso de:

- Antecedente de síndrome intestino irritable, constipación crónica, diarrea crónica, enfermedad celíaca, enfermedad inflamatoria intestinal, esofagitis eosinofílica, gastroenteritis eosinofílica, alergia alimentaria u otra enfermedad gastrointestina crónica

- Antecedente de adenoma de colon o cáncer gastrointestinal

- Antecedente reciente de diarrea o hemorragia digestiva

- Antecedente de cirugía mayor en el aparato digestivo, excluyendo apendicectomía

- Antecedente de enfermedad sistémica autoinmune con compromiso gastrointestinal

- Antecedente de enfermedades neurológicas o neurodegenerativas

- Antecedente de enfermedad psiquiátrica

- Sobrepeso u obesidad (índice de masa corporal > $25 \mathrm{~kg} / \mathrm{m}^{2}$ )

Enfermedades infecciosas. Excluir en caso de:

$\checkmark$ Antecedente de o conocida exposición a virus inmunodeficiencia primaria, virus hepatitis $B$, virus hepatitis $C$, virus humano linfotrófico-T I/II, malaria, tripanosomiasis, tuberculosis

$\checkmark$ Infección sistémica conocida no controlada al momento de la donación

$\checkmark$ Consumo de drogas ilícitas

$\checkmark$ Conductas de riesgo en los últimos 12 meses

$\checkmark$ Ser receptor de un trasplante de órgano o tejido

$\checkmark$ Transfusión de algún producto sanguíneo en los últimos 12 meses

$\checkmark$ Tatuaje o piercing en los últimos 6 meses

$\checkmark$ Reciente ( $<6$ meses) accidente con aguja de jeringa

$\checkmark$ Reciente ( $<6$ meses) antecedente de vacuna con virus vivo atenuado

$\checkmark$ Riesgo de transmisión de enfermedades causadas por priones

$\checkmark$ Viaje en los últimos 6 meses a países con riesgo de enfermedades diarreicas endémicas o diarrea del viajero

$\checkmark$ Trabajo laboral en área de la salud (disminuir riesgo de transmisión de organismos multirresistentes) o área veterinaria (disminuir riesgo de transmisión de infecciones zoonóticas)

\section{Tabla 2. Estudio del donante en caso de programar un trasplante de microbiota fecal} (adaptado de referencias $8,19,21$ ).

Exámenes de sangre:

- Hemograma, proteína C reactiva, velocidad de sedimentación, pruebas hepáticas, creatinina, electrolitos plasmáticos y albúmina

- Virus hepatitis A (anticuerpos IgM)

- Virus hepatitis B (antígeno de superficie y anticuerpos core total)

- Virus hepatitis C (anticuerpos)

- Anticuerpos virus inmundeficiencia humana $(\mathrm{VIH})$

- Anticuerpos virus linfotrópico de células T humanas (HTLV I/II)

- Serología virus Epstein Barr

- Serología citomegalovirus

- Treponema pallidum (VDRL o RPR)

Exámenes de deposiciones:

- Estudio gérmenes enteropatógenos (ideal por reacción de polimerasa en cadena. Opción coprocultivo)

- Clostridium difficile (ideal por reacción de polimerasa en cadena)

- Parasitológico seriado de deposiciones

- Antígeno o tinción para Giardia lamblia, Criptosporidium, Isospora, Cyclospora, microsporidios

- Antígeno Helicobacter pylori 


\section{Resultados}

Durante el período abril 2013-abril 2017, el TMF se ha realizado en 8 pacientes con ICD recurrente, 6 de ellos mujeres (Tabla 3). La mediana de edad de los pacientes fue 48 años (rango 19-82). Siete pacientes habían adquirido la ICD después del uso de antibióticos, la otra paciente tenía el antecedente de haber estado hospitalizada previo a su primer episodio de ICD. Dos pacientes habían usado inhibidores de la bomba de protones previo a la ICD. Un paciente con enfermedad de Crohn se encontraba en tratamiento con azatioprina al momento del TMF. Antes del TMF, todos los pacientes habían recibido al menos dos cursos de dos semanas de tratamiento con metronida- zol o vancomicina y al menos un esquema de vancomicina por plazos prolongados en dosis decreciente o en pulsos. En cuatro pacientes se asoció rifaximina y en uno probióticos. Además, un paciente recibió inmunoglobulina en el episodio previo al trasplante, dada la refractariedad al tratamiento con metronidazol y vancomicina sin tener una respuesta favorable. La mediana de episodios de ICD previo al TMF fue de 4 (rango 3-8). En 4 pacientes la ICD fue grave, necesitando hospitalización, los otros 4 casos fueron leves. El TMF fue realizado, en promedio, 18 días después del inicio del último episodio de ICD (rango 3-36) y la mediana de la duración de la ICD antes del trasplante fue 9 meses (rango 3-18).

El donante fue un familiar directo en seis pa-

Tabla 3. Características demográficas y curso clínico de los pacientes en quienes se realizó un trasplante de microbiota fecal

\begin{tabular}{|c|c|c|c|c|c|c|c|c|c|}
\hline $\begin{array}{l}\text { Paciente } \\
\text { Género / } \\
\text { Edad }\end{array}$ & $\begin{array}{c}\text { Uso de AB } \\
\text { previo a la } \\
\text { ICD }\end{array}$ & $\begin{array}{c}\text { Duración } \\
\text { de la ICD } \\
\text { previo al } \\
\text { TMF } \\
\text { (meses) }\end{array}$ & $\begin{array}{c}\text { n de } \\
\text { episodios } \\
\text { de ICD } \\
\text { previo al } \\
\text { TMF }\end{array}$ & $\begin{array}{l}\text { Severi- } \\
\text { dad de } \\
\text { la ICD }\end{array}$ & $\begin{array}{l}\text { Tratamien- } \\
\text { tos previos } \\
\text { al TMF }\end{array}$ & Donante & $\begin{array}{c}\mathbf{g} \\
\text { heces/ cc } \\
\text { SF }\end{array}$ & $\begin{array}{c}\text { ICD } \\
\text { post-TMF }\end{array}$ & $\begin{array}{l}\text { Segui- } \\
\text { miento } \\
\text { desde el } \\
1^{\text {er }} \text { TMF } \\
\text { (meses) }\end{array}$ \\
\hline $\begin{array}{l}\text { Hombre / } \\
62\end{array}$ & $\begin{array}{l}\text { Claritromicina } \\
\text { Amoxicilina } \\
\text { Ceftriaxona }\end{array}$ & 18 & 8 & Severa ${ }^{\alpha}$ & $\begin{array}{l}\text { Estándar } \\
\text { Rifaximina } \\
\text { Probióticos } \\
\text { Inmuno- } \\
\text { globulina }\end{array}$ & Hija & $69 / 300$ & $\begin{array}{c}\text { ICD post uso } \\
\text { de Ceftriaxona } \\
\text { y Tazobactam } \\
\text { (Tto: Metroni- } \\
\text { dazol/Nancomi- } \\
\text { cina) }\end{array}$ & 55 \\
\hline $\begin{array}{l}\text { Mujer / } \\
57\end{array}$ & Moxifloxacino & 3 & 3 & Severa & Estándar & Sobrino & $150 / 500$ & No & 36 \\
\hline $\begin{array}{l}\text { Mujer / } \\
39\end{array}$ & No & 13 & 5 & Leve & Estándar & $\begin{array}{l}\text { No rela- } \\
\text { cionado }\end{array}$ & $110 / 500$ & No & 36 \\
\hline $\begin{array}{l}\text { Mujer / } \\
34\end{array}$ & Claritromicina & 24 & 8 & Severa & $\begin{array}{l}\text { Estándar } \\
\text { Rifaximina }\end{array}$ & Madre & $100 / 500$ & $\begin{array}{c}\text { ICD sin } \\
\text { antecedente de } \\
\text { AB (Tto: TMF) }\end{array}$ & 31 \\
\hline $\begin{array}{l}\text { Mujer / } \\
35\end{array}$ & $\begin{array}{l}\text { Cefradroxilo } \\
\text { Penicilina }\end{array}$ & 6 & 4 & Leve & $\begin{array}{l}\text { Estándar } \\
\text { Rifaximina }\end{array}$ & $\begin{array}{l}\text { No rela- } \\
\text { cionado }\end{array}$ & $100 / 500$ & No & 17 \\
\hline $\begin{array}{l}\text { Hombre / } \\
19\end{array}$ & $\begin{array}{l}\text { Amoxicilina/ } \\
\text { Ácido } \\
\text { Clavulánico }\end{array}$ & 3 & 3 & Leve & Estándar & Primo & $150 / 500$ & No & 15 \\
\hline $\begin{array}{l}\text { Mujer / } \\
69\end{array}$ & $\begin{array}{l}\text { Amoxicilina/ } \\
\text { Ácido } \\
\text { Clavulánico } \\
\text { Ciprofloxacino } \\
\text { Ceftriaxona }\end{array}$ & 6 & 4 & Leve & $\begin{array}{l}\text { Estándar } \\
\text { Rifaximina }\end{array}$ & Hija & $150 / 500$ & No & 12 \\
\hline $\begin{array}{l}\text { Mujer / } \\
82\end{array}$ & Moxifloxacino & 4 & 3 & Severa & Estándar & Hija & $150 / 500$ & No & 7 \\
\hline
\end{tabular}

AB: antibiótico; ICD: infección por Clostridium difficile; TMF: trasplante de microbiota fecal: SF: suero fisiológico: Tto: tratamiento. ${ }^{\alpha}$ Colitis pseudomembranosa; ${ }^{\beta}$ Tratamiento estándar se refiere a vancomicina y metronidazol. 
cientes y un donante no relacionado en los otros dos. El volumen de heces utilizado en el trasplante fue en promedio $122 \mathrm{ml}$ (rango 69-150) y el promedio de volumen infundido fue $475 \mathrm{ml}$ (rango 300-500). Los hallazgos al momento de realizar el trasplante fueron erosiones en 7 pacientes y divertículos en dos. Un paciente con antecedentes de enfermedad de Crohn y episodios previos de bacteremia por Escherichia coli presentó a las $24 \mathrm{~h}$ del trasplante un nuevo episodio de bacteremia por este microorganismo, siendo tratado con aztreonam, para no afectar de manera considerable la microbiota intestinal postrasplante. La efectividad luego del TMF fue de $100 \%$ y todos los pacientes presentaron una respuesta clínica con deposiciones formadas dentro de la semana. Ningún paciente presentó un nuevo episodio de diarrea dentro de los 6 meses postrasplante. Durante el período de seguimiento (mediana 24 meses, rango 7-55), dos pacientes presentaron una nueva ICD. Uno de ellos fue el paciente con antecedente de enfermedad de Crohn, quien presentó a los 18 meses del TMF un nuevo episodio de ICD después de recibir ceftriaxona y piperacilinia/ tazobactam por bacteremias por Escherichia coli, siendo tratado con vancomicina y metronidazol con buena respuesta. La otra paciente presentó a los 12 meses del TMF, y sin tener el antecedente de uso de antibióticos, un nuevo episodio de ICD realizándose un TMF al momento del diagnóstico con una respuesta clínica favorable. Dos pacientes refirieron la presencia de distensión abdominal y meteorismo posterior al TMF. Todos los pacientes han mencionado que usarían nuevamente esta estrategia en caso de ser necesaria, de hecho, uno de los pacientes decidió utilizarlo como primera estrategia de tratamiento al presentar un nuevo episodio de ICD al año del primer trasplante.

\section{Discusión}

Estudios recientes han demostrado que la ICD recurrente es un cuadro frecuente, que ha aumentado su incidencia en las últimas décadas, de alto costo, con una alta tasa de reingreso hospitalario y con un potencial riesgo de mortalidad ${ }^{25-27}$. La ICD recurrente es un desafío, dado que el tratamiento habitual con cursos prolongados de antibióticos fracasa hasta en $40 \%$ al mantener y exacerbar la disbiosis intestinal ${ }^{28}$. En este escenario, el TMF ha sido sugerido en numerosas publicaciones destacando el Consenso Nacional realizado por la Sociedad de Infectología y Gastroenterología con un nivel de recomendación débil ${ }^{5}$ y el Consenso Europeo publicado recientemente, en que el nivel de recomendación es fuerte ${ }^{9}$, diferencia que estaría dada principalmente por la mayor evidencia actual sobre la efectividad del TMF (81-100\%) ${ }^{17}$. Nuestro estudio confirma estos resultados, dado que todos nuestros pacientes respondieron luego de un TMF. Por otra parte, el trasplante ha sido sugerido en pacientes con ICD refractario con una efectividad que va entre 55 y $100 \%{ }^{9,17,29}$. En nuestro estudio, el primer TMF se realizó en un paciente que había presentado ocho episodios de ICD, siendo el último además refractario a tratamiento antibiótico (vancomicina/metronidazol/ rifaximina) e inmunoglobulina.

En pacientes mayores de 65 años, la ICD está asociada a un mayor riesgo de mortalidad y colectomía. En este contexto, se ha sugerido que el TMF podría ser realizado precozmente después de una segunda recurrencia o después de un primer episodio de ICD grave ${ }^{30,31}$. En nuestra serie, el TMF fue indicado en una paciente de 82 años que presentó dos recurrencias, siendo una de ellas grave necesitando en esa oportunidad hospitalización.

Varias vías de administración para el TMF han sido descritas, incluyendo la endoscopía, sonda nasogástrica, nasoduodenal o nasoyeyunal, enema y colonoscopía ${ }^{17,32}$. Sin embargo, la vía más adecuada para realizar el trasplante no ha sido definida. Aunque algunos autores han señalado que la vía de administración por colonoscopía-enema parece ser más efectiva que por vía alta ${ }^{12,33}$, otros no han demostrado que existan diferencias significativas en la vía utilizada ${ }^{17,34}$. Aunque en nuestro estudio todos los TMF fueron realizados por colonoscopía, creemos que en caso de contraindicación o riesgo de este procedimiento la vía alta es una opción válida.

En relación a la presencia de eventos adversos, la mayoría son leves, autolimitados y de tipo gastrointestinal ${ }^{35}$. Aunque se han descrito complicaciones graves, estas provienen en su mayoría de reportes de casos aislados ${ }^{21}$. Sin duda es necesario contar con estudios con distribución aleatoria y controlados para calificar y cuantificar los riesgos reales del TMF. En nuestra serie, dos pacientes refirieron la presencia de distensión abdominal y meteorismo. Síntomas gastrointestinales después 
del trasplante han sido descritos, los que incluyen náuseas, dolor abdominal, meteorismo, distensión abdominal, cambio en la consistencia y frecuencia de las deposiciones ${ }^{12,16,36}$. Por otra parte, un paciente con enfermedad de Crohn e historia de bacteremias presentó un nuevo episodio por un Escherichia coli $24 \mathrm{~h}$ postrasplante ${ }^{21}$. Es importante considerar que este paciente ha presentado durante su seguimiento de 55 meses post-TMF tres episodios de bacteremias por Escherichia coli, planteando la posibilidad de que el episodio post-TMF no sea debido exclusivamente al procedimiento sino a su enfermedad de base, dado la presencia de un trastorno de permeabilidad intestinal ${ }^{37,38}$. Sin duda, el riesgo de transmitir una infección u otra enfermedad no gastrointestinal desde el donante debe ser minimizado a través de la aplicación de una cuidadosa evaluación de la historia clínica y examen físico del donante y de los exámenes de laboratorio ${ }^{9,39}$. Por otra parte, a pesar de que el TMF ha sido limitado en pacientes inmunosuprimidos debido al teórico riesgo de eventos adversos graves, estudios recientes han demostrado que esta estrategia es segura y efectiva ${ }^{40,41}$. En nuestro estudio, el paciente con enfermedad de Crohn se encontraba en tratamiento con azatioprina al momento de realizar el TMF.

A pesar de la evidencia sobre la efectividad y seguridad del TMF en la ICD recurrente, estudios han demostrado recientemente la subutilización de esta estrategia ${ }^{42}$. Es posible que las características del procedimiento en sí influyan en el equipo tratante y el paciente al momento de evaluar las opciones terapéuticas ${ }^{43,44}$. En nuestro estudio, todos los pacientes mencionaron que usarían nuevamente esta estrategia en caso de ser necesaria, de hecho, un paciente utilizó el TMF al momento del diagnóstico de un nuevo episodio ICD ocurrido un año después de su primer TMF.

Sin duda, las actuales guías y consensos permiten realizar TMF bajo condiciones estándar de selección del donante, preparación de la colonoscopía, utilización de inhibidores de bomba de protones, suspensión de antibióticos, tiempo de obtención de las heces del donante, volumen de dilución y uso de loperamida ${ }^{9,32,33,39}$. En nuestra serie, los pacientes se realizaron el TMF siguiendo las sugerencias dadas, logrando una adecuada efectividad y seguridad en cada uno de los procedimientos.

En relación a la elección del donante, una revi- sión sistemática sugirió que el uso de deposiciones de un donante relacionado al paciente podría ser más efectivo al compararlo con un donante no relacionado (93\% vs $84 \%$ respectivamente) ${ }^{45}$. Sin embargo, otros no han encontrado diferencias significativas de acuerdo al parentesco del donante y el paciente $e^{33}$. Por otra parte, Lee y cols. han sugerido que la utilización de contenido fecal congelado o fresco de donantes universales puede alcanzar idénticos porcentajes de efectividad ${ }^{29}$, siendo una opción al disminuir los costos del estudio del donante y frente a la necesidad de realizar un TMF en pacientes con ICD severa, donde el estudio de un donante relacionado puede significar un retraso en la realización del procedimiento. Además, se ha sugerido que el uso de cápsulas de material fecal congelado puede tener una efectividad de $88 \%$, definido por la ausencia de diarrea en un período de dos meses ${ }^{46}$. Nosotros realizamos el TMF con donante familiar directo en 6 pacientes, los otros dos pacientes eligieron un donante no relacionado, siendo también efectivo.

Finalmente, guías nacionales han sugerido que para evitar resultados falsos positivos es necesario realizar el estudio molecular para ICD en pacientes que presenten un cuadro clínico concordante 2 . En nuestro estudio, todos los pacientes presentaron un cuadro clínico de tres o más deposiciones líquidas en $24 \mathrm{~h}$ antes de realizar el estudio PCR para toxina A y B, evitando de esta manera trasplantar a pacientes colonizados y no necesariamente infectados.

El presente artículo tiene limitaciones, como el pequeño número de pacientes incluidos, ser una experiencia de un único centro y que la cantidad de material trasplantado no fue homogénea en todos los pacientes. En este sentido, guías recientes han sugerido a lo menos un volumen de $30 \mathrm{~g}^{9}$. Con fines de investigación, hubiese sido útil haber podido definir el tipo de cepa de Clostridium difficile o el tipo de microbiota de cada paciente pre y postrasplante y su correlación con el donante durante el seguimiento. Sin embargo, consideramos que nuestro artículo reúne la primera experiencia nacional y muestra la efectividad y seguridad a largo plazo del TMF en pacientes con ICD recurrente.

En conclusión, el TMF vía colonoscopía es una estrategia simple, segura y efectiva, siendo una opción válida en el tratamiento de la ICD refractaria o después de una segunda recaída. Estudios locales idealmente prospectivos y con distribución alea- 
toria podrán definir si la administración por vía alta o el uso de material congelado son igualmente efectivos y seguros en este grupo de pacientes.

\section{Referencias}

1. Bartlett JG, Chang TW, Gurwith M, Gorbach SL, Onderdonk AB. Antibiotic-associated pseudomembranous colitis due to toxin producing clostridia. N Engl J Med 1978; 298: 531-4.

2. Hernández-Rocha C, Naour S, Álvarez-Lobos M, Paredes-Sabja D. [Clostridium difficile associated infections: an update view]. Rev Chilena Infectol 2012; 29: 434-45.

3. Lessa FC, Mu Y, Bamberg WM, Beldavs ZG, Dumyati GK, Dunn JR, et al. Burden of Clostridium difficile infection in the United States. N Engl J Med 2015; 372: 825-34.

4. Magee G, Strauss ME, Thomas SM, Brown H, Baumer D, Broderick KC. Impact of Clostridium difficile-associated diarrhea on acute care length of stay, hospital cost, and readmission: A multicenter retrospective study of inpatients, 2009-2011. Am J Infect Control 2015; 43: 1148-53.

5. Hernández-Rocha C, Pidal P. Ajenjo MC, Quera R, Quintanilla M. Lubascher J, et al. [Chilean consensus of prevention, diagnosis and treatmento of Clostridium difficile-associates diarrhea]. Rev Chilena Infectol 2016; 33: 98-118.

6. Nelson RL, Suda KJ, Evans CT. Antibiotic treatment for Clostridium difficile associated diarrhea in adults. Cochrane Database System Rev 2017; 3: CD004610.

7. McFarland LV, Surawicz CM, Rubin M, Fekety R, Elmer GW, Greenberg RN. Recurrent Clostridium difficile disease: epidemiology and clinical characteristics. Infect Control Hosp Epidemiol 1999; 20: 43-50.

8. Surawicz CM, Brandt LJ, Binion DG, Ananthakrishnan AN, Curry SR, Gilligan PH, et al. Guidelines for diagnosis, treatment and prevention of Clostridium difficile infection. Am J Gastroenterol 2013; 108: 478-98.

9. Cammarota G, Ianiro G, Tilg H, Rajilić-Stojanović M, Kump P, Satokari R, et al. European consensus conference on faecal microbiota transplantation in clinical practice. Gut 2017; 66: 569-80.

10. Khoruts A, Dicksved J, Jansson JK, Sadowsky MJ. Changes in the composition of the human fecal microbiome after bacteriotherapy for recurrent Clostridium difficile-associated diarrhea. J Clin Gastroenterol 2010; 44: 354-360.

11. Eiseman B, Silen W, Bascom GS, Kauvar AJ. Fecal enema as an adjunct in the treatment of pseudomem- branous enterocolitis. Surgery 1958; 44: 854-9.

12. van Nood E, Vrieze A, Nieuwdorp M, Fuentes S, Zoetendal EG, de vos WM, et al. Duodenal infusion of donor feces for recurrent Clostridium difficile. N Engl J Med 2013; 368: 407-15.

13. Youngster I, Sauk J, Pindar C. Fecal microbiota transplant for relapsing Clostridium difficile infection using a frozen inoculum from unrelated donors: a randomized, open-label controlled pilot study. Clin Infect Dis 2014; 58: 1515-22.

14. Cammarota G, Masucci L, Ianiro G, Bibbo S, Dinoi G, Costamagna $G$, et al. Randomised clinical trial: faecal microbiota transplantation by colonoscopy vs vancomycin for the treatment of recurrent Clostridium difficile infection. Aliment Pharmacol Ther 2015; 41: 835-43.

15. Lee CH, Steiner T, Petrof EO, Smieja M, Roscoe D, Nematallah A, et al. Frozen vs fresh fecal microbiota transplantation and clinical resolution of diarrhea in patients with recurrent Clostridium difficile infection: a randomized clinical trial. JAMA 2016; 325: 142-9.

16. Kelly CR, Khoruts A, Staley C, Sadowsky MJ, Abd M, Alani M, et al. Effect fecal microbiota transplantation on recurrence in multiply recurrent Clostridium difficile infection. Ann Intern Med 2016; 165: 609-16.

17. Quraishi MN, Widlak M, Bhala N, Moore D, Price M, Sharma N, et al. Systematic review with meta-analysis: the efficacy of faecal microbiota transplantation for the treatment of recurrent and refractory Clostridium difficile infection. Aliment Pharmacol Ther 2017; 46: 479-93.

18. Weingarden A, González A, Vásquez-Baeza Y, Weiss S, Humphry G, Berg-Lyons D, et al. Dynamic changes in short-and long-therm bacterial composition following fecal microbiota transplantation for recurrent Clostridium difficile infection. Microbiome 2015; 3: 10.

19. Baxter M, Ahmad T, Colville A, Sheridan R. Fatal aspiration pneumonia as a complication of fecal microbiota transplant. Clin Infect Dis 2015; 61: 136-7.

20. Khan MA, Sofi AA, Ahmad U, Alaradi O, Khan AR, Hammad T, et al. Efficacy and safety of, and patient satisfaction with, colonoscopic-administered fecal microbiota transplantation in relapsing and refractory community- and hospital-acquired Clostridium difficile infection. Can J Gastroenterol Hepatol 2014; 28: 434-8.

21. Quera R, Espinoza R, Estay C, Rivera D. Bacteremia as an adverse event of fecal microbiota transplantation in a patient with Crohn's disease and recurrent Clostridium difficile infection. J Crohn's Colitis 2014; 8: 252-3.

22. Moscoso F, Hevia M, Quera R. Trasplante de microbiota fecal en paciente con colitis ulcerosa e infección refractaria por Clostridium difficile: revisión de la literatura a 
partir de un caso clínico. Rev Gastroenterol Latinoam 2014; 25: 275-81.

23. Moscoso F, Simian D, Rivera D, Acuña G, Quera R. Trasplante de microbiota fecal en infección recurrente por Clostridium difficile. Experiencia local a partir de un caso clínico. Rev Med Chile 2015; 143: 531-5.

24. Hernández-Rocha C, Tejos R, Plaza-Garrido Á, Barra-Carrasco J, Agüero G, Inostroza G, et al. [Performance of prognostic index in severe Clostridium difficile associated infection: retrospective analysis in a universital hospital]. Rev Chilena Infectol 2014; 31: 659-65.

25. Ma GK, Bresinger CM, Wu Q and Lewis JD. Increasing incidence of multiply recurrent Clostridium difficile infection in the United States. Ann Intern Med 2017; 167: 152-8.

26. Olsen MA, Yan Y, Reske KA, Zilberberg M, Dubberke ER. Impact of Clostridium difficile recurrence on hospital readmissions. Am J Infect Control 2015; 43: 318-22.

27. Olsen MA, Yan Y, Reske KA, Zilberberg M, Dubberke ER. Recurrent Clostridium difficile infection is associated with increased mortality. Clin Microbiol Infect 2015; 21: 164-70.

28. Chang JY, Antonopoulos DA, Kalra A, Tonelli A, Khalife WT, Schmidt TM, et al. Decreased diversity of the fecal microbiome in recurrent Clostridium difficile-associated diarrhea. J Infect Dis 2008; 197: 435-8.

29. Lee CH, Steiner T, Petrof EO, Smieja M, Roscoe D, Nematallah A, et al. Frozen vs fresh Fecal Microbiota Transplantation and Clinical resolution of diarrhea in patients with recurrent Clostridium difficile infection: A randomized clinical trial. JAMA 2016; 315: 142-9.

30. Cheng YW, Fischer M. The present status of Fecal Microbiota Transplantation and its value in the elderly. Curr Treat Options Gastroenterol 2016 Jul 26 doi.

31. Agrawal M, Aroniasis OC, Brandt LJ, Kelly C, Freeman $\mathrm{S}$, Surawicz $\mathrm{C}$, et al. The long-therm efficacy and safety of Fecal Microbiota Transplantation for recurrent, severe, and complicated Clostridium difficile infection in 146 elderly individuals. J Clin Gastroenterol 2016; 50: 403-7.

32. Brandt LJ, Aroniadis OC. An overview of fecal microbiota transplantation: techniques, indications, and outcomes. Gastrointest Endosc 2013; 78: 240-9.

33. Smits LP, Bouter KE, de Vos WM, Borody TJ, Nieuwdorp M. Therapeutic potential of fecal microbiota transplantation. Gastroenterology 2013; 145: 946-53.

34. Postigo R, Kim JH. Colonoscopic versus nasogastric fecal transplantation for the treatment of Clostridium difficile infection: a review and pooled analysis. Infection 2012; 40: 643-8.

35. Baxter M, Colville A. Adverse events in faecal microbiota transplant: a review of the literature. J Hosp Infect 2016; 92: 117-27.

36. Youngster I, Sauk J, Pindar C, Wilson RG, Kaplan JL, Smith MB, et al. Fecal microbiota transplant or relapsing Clostridium difficile infection using a frozen inoculum from unrelated donors: a randomized, open-label controlled pilot study. Clin Infect Dis 2014; 58: 1515-22.

37. Vrakas S, Mountzouris KC, Michalopoulos G, Karamanolis G, Papatheodoridis G, Tzathas C, et al. Intestinal bacterial composition and translocation of bacteria in Inflammatory Bowel Disease. PLoS One 2017; 12: e0170034.

38. Teshima CW, Dieleman LA, Meddings JB. Abnormal intestinal permeability in Crohns'disease pathogenesis. Ann N Y Acad Sci 2012; 1258: 159-65.

39. Sokol H, Galperine T, Kapel N, Bourlioux P, Seksik P, Barbut $\mathrm{F}$, et al. Faecal microbiota transplantation in recurrent Clostridium difficile infection: Recommendations from the French Group of Faecal Microbiota Transplantation. Dig Liver Dis 2016; 48: 242-7.

40. Webb BJ, Brunner A, Ford CD, Gazdik MA, Petersen FB, Hoda D. Fecal microbiota transplantation for recurrent infection in hematopoietic stem cell transplant recipients. Transpl Infect Dis 2016; 18: 628-33.

41. Alrabaa S, Jariwala R, Zeitler K, Montero J. Fecal microbiota transplantation outcomes in immuncompetent and immunocompromised patients: A single-centre experience. Transpl Infect Dis 2017 Aug;19.doi

42. Prior AR, Kevans D, McDowell L, Cudmore S, Fitzpatrick F. Treatment of Clostridium difficile infection: a national survey of clinical recommendations and the use of Faecal Microbiota Transplantation. J Hosp Infect 2017; 95: 438-41.

43. Zipursky JS, Sidorsky TI, Freedman CA, Sidorsky MN, Kirkland KB. Patient attitudes toward the use of fecal microbiota transplantation in the treatment of recurrent Clostridium difficile infection. Clin Infect Dis2012; 55: 1652-8.

44. Zipursky JS, Sidorsky TI, Freedman CA, Sidorsky MN, Kirkland KB. Physician attitudes toward the use of fecal microbiota transplantation for the treatment of recurrent Clostridium difficile infection. Can J Gastroenterol Hepatol 2014; 28: 319-24.

45. Gough E, Shaikh H, Manges AR. Systematic review of Intestinal Microbiota Transplantation (fecal bacteriotherapy) for recurrent Clostridium difficile infection. Clin Infect Dis 2011; 53: 994-1002.

46. Staley C, Hamilton MJ, Vaughn BP, Graiziger CT, Newman KM, Kabaje AJ. Successful resolution of recurrent Clostridium difficile infection using freeze, dried, encapsulated fecal microbiota; pragmatic cohort study. Am J Gastroenterol 2017; 112: 940-7. 